\title{
Climatic processes and agriculture
}

\section{B. Panasiuk,}

\author{
Academician of the NAAS, Doctor of Economic Sciences \\ Institute of Bioenergetic Crops and Sugar Beet of the NAAS
}

The purpose. To study and justify dependence of agriculture on new climatic processes on an instance of separate components, in particular dynamics of soil moisture and saturation with carbon dioxide $\left(\mathrm{CO}_{2}\right)$. Methods. Analytical, statistical, generalizing philosophical-scientific understanding of nature of wildlife and climate; dependence of photosynthesis on natural instability of climatic processes, humanitarian-scientific understanding of sunspace energy. Results. It is proved that in modern conditions, together with rainfall and temperature of air, growth of cultivated plants is considerably depends on carbon dioxide $\left(\mathrm{CO}_{2}\right)$ and soil moisture. Conclusions. To determine perspectives of further development of agriculture it is necessary to use in an index of hydrothermal quotient (HTQ) parameters of long-term daily average rainfall and temperatures of air, parameters of humidity of soils and level of concentration of carbon dioxide $\left(\mathrm{CO}_{2}\right)$ which are expedient for fixing in the certain terrain.

Key words: photosynthesis, soil moisture, carbon dioxide $\left(\mathrm{CO}_{2}\right)$, rainfall, temperature of air, hydrothermal quotient, crops.

Modern geological science claims that plants as representatives of living matter first appeared in the water, forming oxygen and the ozone layer. Subsequently, on the land began to form a modern plant and animal world, this process continues to this day. Often the question arises: Is it really that, having originated 3.8 billion years ago in the form of the simplest unicellular bacteria, has life been formed for so long from simple to complex? After all, on the planet Earth complicated, sometimes terrible geological processes occurred, the planet changed from one orbit to another, the climate changed many times.

Even if we take into account modern research and conclusions that after the formation of our Galaxy Galaxy, its components, many of which are planets, stars and other objects, are moving away from one another [28], it can be assumed that our planet Earth in the future will either move to another orbit, or move away from the Sun. In this case, the sun's rays can not create the proper temperature conditions for the plant leaves to absorb the spores that will come from the expanses of the Universe. And there will be no plants - there will be no life, because life can exist on the planet Earth thanks to "continuously and, apparently, invariably during the geological time, the inflow of cosmic energy, mainly the radiant energy of the Sun" [6].

Given this, it is advisable to revise the scientific foundations of the geological history of planet Earth, recognizing that the planet has been subjected and still experiencing cardinal climatic processes due to changes in poles, and possibly orbits, according to some scientists, and in every geological epoch. new manifestation of living matter (the aggregate of living organisms) in accordance with the external environment. If this is not done, then modern humanity will never know about living matter and its manifestation on the planets of the solar system and the universe as a whole. First of all, we must raise the question of how and when the living matter manifested itself in the modern geological epoch on our planet. We offer our own concept, confirmed by scientific studies of outstanding scientists of the world, both past and present.

Living matter and life is the foundation of the existence of plant and animal life and human civilization in general. Today, there is every reason to recognize the possibility of significant climate change, both in general on the planet Earth, and on every continent, in every country in particular. Possible climatic changes feel the living matter - nature, plant and animal world, because "... The characteristic property of a living substance is its variability, its ability to adapt to the conditions of the external environment, which includes" temperature, pressure, phase of the medium, chemistry of the medium, radiant energy "[2], and therefore the appearance of a new in the external environment is of practical interest. 
That is why the knowledge of living matter and the mystery of life constantly excites humanity, this is the focus of research of philosophers, theologians, scientists, artists. Successes are scanty, there are many examples, when investigating the problem of the mystery of life, scientists admitted that "... science has not given its entire history - more than two and a half thousand years - no answer to this everstanding question before us ... science still looking for ways for this decision "[3]. At the same time, scientists do not lose hope, and answering the question: "Has there ever been a space without manifestations of life, can there be a lifeless Universe?" Give an optimistic answer: "We know - and we know scientifically, that the space without matter and without energy can not exist "[4]. To solve the mystery, science even resorts to the reproduction of living organisms from synthetic biology, which somewhat smooths the difference between living and inanimate, the concept of "abiogenesis" emerges from inanimate. However, when it comes to the results of the production of living matter, then we hear: "Whether it will be or not, it is difficult to say" [6].

Continuing the search for unknown forces of nature that determine the existence or at least the manifestation of life, science has come to the conclusion that physical, chemical, mechanical processes do not provide an opportunity to understand this mystery: "The trace of ancestors is lost in the darkness of the unknown. Their forms, organisms were different than ours. ... Their past existence is not a fiction "[7]. It may be true that the main reason for the failure of the millennial search for the solution of the "mystery of life" is the non-recognition by science of the existence of material and immaterial in living matter, man and the Universe. No wonder the great thinker V. Vernadsky, having conducted the research, wrote: "I was convinced of the extreme scarcity, incompleteness and randomness of our knowledge about the volumes of chemical composition, weight and energy of organisms." The scientist saw some imperceptible essence that exists in the body, but which is difficult to recognize, and therefore he set himself a secret question ahead of time: "Is there really a special life energy that obeys the same laws that govern all others?" [8].

Referring to the fact that science is in search of ways to cognize the mysteries of life, let us give some results of our own research on such an important problem - living matter and life. First of all, we should agree that since the existence of the material and non-material world is not recognized, it is not yet time to investigate the appearance of the animal world, including man, However, some research results, in the future, pave the way to the truth, we can cite.

What, besides the sun's rays, thanks to photosynthesis promotes the production of organic matter the basis of life on the planet Earth? It is because of the above-mentioned reason that the science and practice of the countries of the world now also uses such a definition of photosynthesis in which there is no other element of the formation of living matter, except for sun rays and chlorophyll: "Photosynthesis is the transformation of the sun's radiant energy into energy by green plants and photosynthetic microorganisms chemical bonds of organic substances. It occurs with the participation of light absorbing pigments (chlorophyll, etc.). Photosynthesis is the only biological process that proceeds with an increase in free energy and directly or indirectly provides all terrestrial organisms with accessible chemical energy, "wrote K. Timiryazev.

First of all, one important conclusion must be drawn from known studies, to which modern science has never reached the complex: in the water, life first appeared (perhaps there it appeared much later), but only green plants, algae, capable of perceiving the sun's rays, and water, which forms carbon dioxide and produces hydrogen, oxygen and carbon. The carbon dioxide dissolves, the oxygen released by the leaves enters the air, the plant consumes carbon, which is the basis of the organic matter and constitutes an important part (45\%) of the plant itself. Thus, the most important substances for plants come from air and water. This is how the air of our planet was filled with oxygen, when the first simple plants appeared in water and on water. "Thanks to photosynthetic activity of the first green organisms, oxygen appeared in the primary atmosphere of the Earth, an ozone screen appeared, conditions for biological evolution were created" [36].

When oxygen was formed in sufficient quantities, the ozone layer formed, then plants appeared on land. The plant world formed a favorable external environment for the appearance of the animal world, including man. Perhaps, most likely, that the primary source - the basis of all life on Earth - looks like it was described by K. Timiryazev: "All organic substances, no matter how diverse they are, wherever they 
are found, whether in a plant, in an animal or a person, have passed through a leaf, have come from substances produced by a sheet. Outside the leaf, or rather, outside the chlorophyll grain in nature, there is no laboratory where organic matter is excreted. In all other organs and organisms it is transformed, transformed, only here it is formed again from the substance of the inorganic "[38]. However, here a number of questions arise that require an explanation.

However, K. Timiryazev never came to the only correct opinion, from which the organic substance is produced in the chlorophyll grain of the leaf during photosynthesis (except water, carbon dioxide, oxygen, carbon and sun rays) in order to obtain starch in the end. "But how it happens; where does oxygen come from, whether from carbon dioxide or partially from water, which is even likely, and does not precede the formation of starch formation of other simpler, and perhaps more complex compounds - we do not know exactly what this is, and there is no place ... interpretation of these phenomena, not yet fully explained by science "[39]. Such a statement by K. Timiryazev did not come about by chance, since the well-known scientist felt that there was insufficient understanding of the other components involved in the creation of living matter in the laboratory-a sheet. Perhaps he knew about the teachings of S. Arrhenius (1859-1922), who also had predecessors-like-minded people who spoke about the probability of the arrival on the planet of Earth of special parts of cosmic dust that brings the germ of living matter to leaflets, but did not draw this is enough attention. Therefore, the great Russian scientist has left his doubts to the descendants and the mysteries of the processes occurring in the leaves of plants are not fully understood. Later, science shed light on many things, but "other, simpler, and perhaps more complex compounds," apparently did not fully explain.

Referring to the teachings of $\mathrm{S}$. Arrhenius and his predecessors, we can at least hypothetically assert that spores of cosmic dust (the germ of living matter), that enter our planet so quickly that the ultraviolet rays of the sun do not destroy them [9] . V. Vernadsky recalled the possible existence of the dispute. In his opinion: "The spiral nebula of the Galaxy, i.e. our galaxy, has the form of a colossal lens, in the vacuum of which the material-energy body-ions, free atoms and molecules, cosmic dust, meteorites, comets, planets, stars are scattered. The great merit of $\mathrm{S}$. Arrhenius was the establishment that among the dust there must be countless disputes - the embryos of living matter that emanate from the planet, the terrestrial planets, at least, and again they fall into the course of time "[10].

In the light of the teachings of well-known philosophers and scientists, there is reason to at least hypothetically assert that it is not the sun's rays that supply some kind of a living substance to the planet, but they (the rays-BP) only create conditions for the assimilation of each sheet of the dispute that come from outer space. Disputes like embryos of living matter are part of cosmic dust, and therefore exist outside the sun's rays and according to the laws of the universe they come to the planets of the Universe, where favorable conditions are created, in particular for the planet Earth. That is why scientists noticed not only a complex feature of the structure of the leaf, but also its location on plants, color and the presence in the leaf of a special natural laboratory where the inorganic substance turns into organic.

No wonder nature has given the leaves of plants certain features, in particular, placing each leaf of any tree at such a distance that none of them obscures the other from the sun's rays. Leaves of all plants are located in accordance with the golden section $1+5=1,618033988$, provide each leaf with internal hidden forces that are transmitted to all living things - both the plant itself and the animal world. Everything created in the leaves of plants is used by the organisms of the biosphere, which are numerous and which have certain characteristics.

So, there is a high probability that nature has given the leaf the opportunity to perceive the spores that constantly come from outer space and other planets of our solar system. However, the leaflet can assimilate them for transformation into organic matter at certain favorable temperatures, which must be created by the sun's rays. In winter, when there is no heat, the tree discards leaves, which is already superfluous, and therefore "not a leaf as a whole, namely, the chlorophyll grain that informs it green ... serves as a mediator between all life on Earth and the Sun" [40]. So it is proved that living matter or its form comes from outer space, however, yet what comes from outer space? V. Vernadsky tried to answer this question: "Since no one has ever been able to prove the existence of the smallest simple elements of the organism scattered in Nature outside the organism, and even less - of simple organized forms of matter, not living and not dead, but some intermediate, it was not possible to get these organisms and 
their morphologically to study, the only real proof of their existence was their manifestation in the environment, i.e. in the earth's crust - this way it is proved the existence of organisms smaller than the length of light waves, and inaccessible to the eye "[11].

Such thoughts existed in the twentieth century, when the central place in biological science was occupied by the theory of G. Mendel (1869), according to which genes control and influence the entire course of the development of plant and animal organisms from birth to death. It was alleged that the main force determining the trends of evolution of all life on Earth is the gene. However, scientists of the XXI century. (M. Roik - Ukraine, S. Maletsky and V. Dragavtsev - Russia) investigated this issue and came to the conclusion: "In our opinion, parthenogenetic development of embryos in plants ... is associated with the production by cells of embryonic sacks or ovules of a flower of external or internal signals that allow one to move from one development program to another" [35]. That is, genetic scientists recognize that the genetic chain in the cell is exposed to external influences. If we take into account that the consciousness, as well as the unknown influence on the living nature is an immaterial constant, the question arises: what forces have given the person consciousness, what influences the bacterium and cell? The answer to this question will concern living matter, of course, of man, as well as of any nonmaterial energy. So, the energy of the Universe carries information (signals), exerting an external influence on the cell, whose genes also fulfill their role as an instrument, that is what V. Vernadsky thought about it.

Referring to the ideas of outstanding scientists about living matter, let us define the concept of "living matter": the living substance is physical, chemical and mechanical forces that are rapidly and unnoticed, constantly supplemented by the vital energy of the Universe, which determines the weight and energy of organisms. By physical, chemical and mechanical forces we mean a certain number of chemical elements entering and leaving through a living organism, in particular, silicon, aluminum, titanium, copper, zinc, lead, silver, rubidium and others, which are a form of matter. Under the vital energy of the Universe we understand the germs of living matter that come from space in the form of spores of cosmic dust - this is a form of energy. So, we have 2 forms of cosmic manifestation of the living on the planet Earth. The third form of manifestation of life - the spiritual principle - is not yet recognized by science, except for individual scientists. Such an understanding of living matter corresponds to the most important feature of the living organism - the constituent of the cosmic indivisible, this was emphasized by Cuvier (1769 1832) and Academician Vernadsky [12].

Considering that "Man - an animal is a social, heterotrophic. It can exist only under condition of the existence of second organisms, namely, green plants "[13], allow ourselves to make a series of conclusions and assumptions with comments, statements and objections regarding certain studies.

First. In outer space, there is life (or elements for living nature), it is eternal, as the universe in space and time. The same opinion, citing Aristotle, Redi, Richter, Arrhenius, and others, was also maintained by V. Vernadsky: "For us it becomes clear that life is a cosmic phenomenon, and not specifically earthly" [14]. So, life must be sought there and expected from there. It is from outer space that life comes to the planets of solar systems, at a time when favorable conditions are created for the birth of the plant and animal world, including man.

Humanity is interested in the problem of the appearance of a human being, for only then can it know about the appearance of the plant and the entire animal world. Some of the modern researchers believe that the appearance of living organisms took place in a certain geological period. "At a certain distance from the Sun, the atmosphere and water appear on the planet. ... After the stabilization of the new parameters, a compulsory launch in the construction of biological organisms at the cell level takes place "[29]. Perhaps this is so, but what is this design: there is abiogenesis, archeogenesis, heterogenesis or biogenesis is unknown, it is only a general mental statement.

However, representatives of the science of the past held the view that the emergence of life can only be represented empirically, "The beginning of which is lost to us ... No scientific theory has hitherto been able to embrace, as a whole, the paleontological evolution of organized beings, the last important manifestation of which was the emergence of man" [15].

There is every reason to suppose that for the billion-year geological epoch (2.6 billion years) of the planet Earth, when the favorable conditions for the emergence of life disappeared and restored, so each 
time after climate change a special climate environment was formed, which contributed to the emergence of new manifestations of living nature. However, perhaps it, that is, a qualitatively new nature, the plant and animal world, as well as man, were not similar to the present. Everything was different - another air temperature in the direction of decline, other plants, everything is qualitatively different. Philosophers, scientists and physicists are coming closer to the point when it will be possible to say that during the geological history of the Earth there were other civilizations, but between them "there was no continuity or heredity, no internal connections between civilizations and their cultures exist, each of they appear and disappear separately "[30]. So, if we talk about the modern man, then it will be justly asserted that he appeared once for the entire geological history of the Earth, but there were other periods, other animals, other plants, other people that we do not even know about.

The second. Life can not be transferred from the planet to the planet, it exists in the Cosmos as an integral part of living matter. Life is fed to the planet Earth, as to other planets of the Cosmos, constantly through the leaves of plants. "To the person as the recipient from the primary source, the Creator, information and energy come through plants and wave oscillations" [31]. On the planet Earth constantly and massively enter disputes as the basis of life, they are assimilated by leaves of plants. The solar rays only contribute to the process of assimilation, creating a favorable external temperature, the external environment. "The problem of the beginning of life is the problem of the beginning of the life environment on our planet. ... A solid monolith (life environment) was created, and not separate species of living organisms "[16]. That is, on the planet conditions are created first, and then life arises. The person gradually learns the conditions of life, but for the origin of life he can not even come close. First of all, scientists, in particular V. Vernadsky, believed that "Cosmic matter constantly in different forms gets to Earth, and the earth goes into outer space" [17]. So, the scientist believed that when a living substance comes out of a particular planet, it gets to outer space, and this does not mean that from the planet to the planet. In this connection, there is a doubt about the existence of the transmission of living matter from the planet to the planet, and therefore "Panspermia - the hypothesis of the possibility of transferring life in the universe from one cosmic body to another" [37], at least is in doubt for a number of reasons. For example, V. Vernadsky himself did not take it very much, he had doubts: "For thousands of generations there is a mystery unresolved, but as if resolved - the mystery of life. This is the mystery of life for us, but not in the reality surrounding us. It indicates only that we do not understand reality "[18], therefore, we do not understand something. In addition, if at least one scientist had proved that the living matter had been transferred from planet Earth to another planet, or something similar had happened to our planet, but this is not. The same $\mathrm{V}$. Vernadsky wrote: "The existence of such an entry has not been anywhere and never scientifically established," [19]. There are other reasons. In our opinion, such transfers are also impossible, because "if we assume the existence of life on other planets, then there are possible other hypotheses of development laid down by the Higher Reason" [32], and therefore such a transfer is not only inexpedient, but impossible.

Third. From the above, it follows that the appearance of life on the planet Earth is directly related to the temperature conditions in the water and on land created by the Sun and the planet itself for the development of the plant world, which, in turn, receives living substance from life through its leaves. Life appears in certain periods of geological history of the Earth, when in a few places - in water or on the surface of the Earth, conditions are created for a kind of "font", where living organisms are born.

Here we only note that the sun's rays do not carry living matter, that is, life, but only give energy to the plant and animal world. When the biological life-span of a plant and an animal ends, they form an inanimate nature - coal, gas, oil, iron ore, coral reefs, etc. They accumulated solar energy, which now man uses for his own needs, but living matter in a once living nature has already no. So, the Sun and its rays carry only energy to the planet Earth, life enters the vegetable and animal world from outer space in a different way, as discussed above.

Fourth. It can be assumed that life has already occurred on the planets of Mars and Jupiter, has faded and has now disappeared as a result of the distance from the Sun, and on the planets of Mercury and Venus, life may arise in the future if they are separated from the Sun by a distance at which the sun's rays create conditions favorable for the acceptance of plant leaves by the dispute of life from outer space. Venus is the next planet after Earth, where, perhaps, there will be a life that on Earth can disappear. On 
Mars, life was already, it disappeared and appeared on Earth, now it's up to Venus. As soon as Venus has favorable climatic conditions, life will appear there, because living matter (life) exists in the Universe everywhere and always. "Life is eternal in so far as the cosmos is eternal" [20].

Fifth. Disputes that fill the universe, the only ones, but the climate on each planet in different periods of their geological path is different. It is the climate that creates the conditions under which a plant world develops on a particular planet with a division of plants for the tropics, the north, the south, the temperate and arid belts. This gives grounds to confirm once again the determining role of climate and its dynamics for living nature on the planets of the Universe. That is why it is legitimate to say that life exists both on other planets of our solar system and in other solar systems of our Galaxy, because "What is true for tens and hundreds of millions of years ... cosmic periods of the history of the Earth, is true for the whole universe" [21].

So, in our opinion, a living substance appeared on planet Earth, as well as on other planets of the Universe. Now science does not know how and where the animal world appeared, including a human being, but let us try to state some considerations that will bring us closer to the knowledge of this mystery by a miserable iota. Each species of plants and animals, including man, is born from living matter at the same time, but separately and with a certain order: first the plant world appears, then an animal, including man, arises and develops on the basis of the plant world. The appearance of the animal world, including humans, 2.6 million years ago, is a manifestation of wildlife, as discussed above.

The first, in our opinion, must be recognized that each species of plant and animal life, including man, appears in a corresponding geological era separately in accordance with the new climatic conditions, and therefore each species has its own history of beginning and end as the basic law of nature and the universe. The proof of the above statement is that every plant, animal, just like a human, has a certain facet of existence. "We are not able to explain to ourselves the necessity of such a process, we are not able to understand why one and the same plant could not exist indefinitely" [22].

Over the entire geological history of the planet Earth, a living substance that exists in outer space has manifested itself more than once: as a result of climatic changes, some plants and animals (for example, dinosaurs) disappeared, others appeared. Not bypassed, of course, this is such a representative of the animal world as a man, although he is a special animal endowed with reason. It can not be denied that animals appear, disappear, and reappear, but they are completely different, but man has remained unchanged in all ages, despite the transition of the planet to another orbit, to replace the climate. Knowing how fast the processes in the leaf laboratory work, science for some reason has long been allocating billions and millions of years for the formation of cells of living nature, for the emergence of the same person - less time (5 - 1.8 million years).

An important proof that plants, animals and humans appear independently, and do not originate from each other, is also the fact that each plant and animal has its own geochemical energy, which determines the speed of reproduction of each species. For example, an elephant, "It propagates in the biphospher at a rate that does not exceed a fraction of a millimeter of a second. The same is true of man "[23]. But there are many medium, small and small animals, microbes, and in the case when there is a question about the origin of the animal world from one person, it is necessary to prove that nature, laws of the Universe make it possible to change the energy of development and reproduction for each individual in the process of its separation from some kind of unified energy. This can not be proved, since the laws of the universe do not change, they are constant, and therefore we reaffirm that every species of plant and animal life, including man, has appeared separately, has its own history, genetics, its material and immaterial nature.

If we admit that each species of the animal world will appear on the planet Earth once for the geological history, will have a beginning and an end, then when it disappears, a completely different appearance will appear, unlike the previous one. For example, mammoths, dinosaurs and other species will not appear again. If we consider the timing and process of the appearance of certain species of the animal world, then it is possible to consider the following: first, the term of occurrence is a short interval of geological time, similar to the term of the birth of any animals, including humans; second, the process of appearance arises in a natural environment, when favorable conditions are created for a living substance capable of perceiving embryos of life coming from outer space in the form of spores or other manifestations. From the scientific and philosophical point of view, the appearance of life is not an 
accident, but a natural manifestation of the laws of the universe, this is the creation of special conditions in a certain geological period of the history of the planet Earth for the manifestation of the genesis of life.

So, the period of existence for each species of the animal world and man is the same in one or another geological era, and the period of origin corresponds to the phenomenon of biogenesis "Like the way we see it around, when a person and other organisms are born from other living beings, besides their own kind" [24]. At the same time, the process of appearance corresponds to the influence of such two phenomena - abiogenesis (origination outside the living one) and archeogenesis (initial nucleation). This statement corresponds to the scientific tradition of the researchers of the past: Pouchet (1800 - 1872), his supporters - Bastian, Boyshan and their followers, whose scientific idea confirms the thesis that living matter "is carried into the smallest manifestations of life, into more elementary bodies than cells-into microsomes, gemmules, and so on. - phenomena of heterogeneity or abiogenesis "[25].

Attempts to use synthetic biology to reproduce life from a dead nature in terms of composition and form of a living organism can not be achieved, since synthetics is not a living substance in which there exists "Invisible ... world of microscopic beings" [26], capable of receiving and combining with elements of life coming from outer space. Yes, we see that the world of microorganisms is everywhere - in water, air, soil, dust, microorganisms are constantly manifested during decay and fermentation, it is in them that there are imperfect organisms - microtomes, bacteria, etc., outside the cell, which "are released from the contents of cells upon their destruction, are capable in some cases of existence after the death of cells and the death of the organism composed of them" [27]. Apparently, as has already been said, these socalled imperfect organisms, under favorable conditions in a certain geological era, receiving embryos of life from outer space, give rise to the origin of the plant and animal world, including man. We have already referred to the words of outstanding scientists that the universe can not exist without living matter, without life, therefore the existence of organisms outside the cell on the planet Earth, which is a grain of sand in the space of the universe, just confirms the evidence given above.

When science proves the beginning of the appearance of man, and, perhaps, the secrets of its appearance are recognized, there will remain another great mystery: how, for what, and what power has man endowed with reason? But this person probably never will understand, and does not investigate this is the mystery of secrets, although there are some unproven reasonings of some researchers, in particular L. Gumilev, D. Chizhevsky, A. Lazarev.

It is the mind that nature has given to man that determines the role of man on the planet Earth. In our opinion, a person has the main task - to serve as a transmitter of what is happening on the planet, to the Universal Mind. This happens through the WIS (Information System of the Universe), which "is a cosmic connection ... through the information field that is contained in the interplanetary medium" [33]. Perhaps, there is no other function, since the research of many scientists shows that a person uses the external environment with his living and inanimate substance not only to build his body, as does the whole animal world, but also for the high needs of his social life, which is a powerful geological process.

This process is gaining momentum, people have taken possession of all living matter, especially plants, which determine the fate of all other living matter, as well as the animal world to which he himself belongs. So, a person uses the living substance of the planet Earth on his own. Using the received mind, it mainly harms the nature and itself, as well as the whole animal world, which nature can not afford in its essence, and if this process is not stopped or at least not ordered, then, as Vernadsky warned, "a person will go to end if he does not meet with an opposing external force that will destroy it or keep it in a potentially state. " In our opinion, perhaps this will not happen, which should be reassured by mankind, since "nature has subordinated man's willful decisions to the force of necessity" [34], which will restrain his negative actions.

\section{Conclusions}

To determine perspectives of further development of agriculture it is necessary to use in an index of hydrothermal quotient (HTQ) parameters of long-term daily average rainfall and temperatures of air, parameters of humidity of soils and level of concentration of carbon dioxide $\left(\mathrm{CO}_{2}\right)$ which are expedient for fixing in the certain terrain. 


\section{References}

1. Weber Bernard. Encyclopedia Relative to absolute knowledge. - M.: Ripol the classic, 2009. - P. 102.

2. Selected scientific works of VI. Vernadsky. - T.

4. The book. 1. - M., 2012. - P. 288.

3. Ibid. - P. 34 - 35.

4. Ibid. - P. 33.

5. Ibid. - P. 25.

6. Selected scientific works of Academician Vernadsky. - T. 4. Prince. 2. - M., 2012. - P. 439.

7. Selected scientific works of Academician V. Vernadsky. - T. 4. Prince. 1. - M., 2012. - P. 116.

8. Ibid. - P. 26 - 27.

9. Selected scientific works of Academician V. Vernadsky. - T. 3. - M., 2012. - P. 70.

10. Ibid.

11. Selected scientific works of Academician V. Vernadsky. - T. 4. Prince. 1. - M., 2012. - P. 116.

12. Ibid. - P. 33.

13. Ibid. - P. 122.

14. Ibid. - P. 55.

15. Ibid. - P. 122.

16. Ibid. - P. 443.

17. Ibid. - P. 55.

18. Selected scientific works of Academician V. Vernadsky. - T. 3. - M., 2012. - P. 237.

19. Selected scientific works of Academician V. Vernadsky. - T. 4. Prince. 1. - M., 2012. - P. 54.

20. Ibid. - P. 52.

21. Ibid.

22. Selected scientific works of Academician V. Vernadsky. - M., 2012. - T. 3. - P. 249.

23. Selected scientific works of Academician V. Vernadsky. - M., 2012. - T. 4. Prince. 1. - P. 167.

24. Ibid. - P. 36.

25. Ibid. - P. 46.

26. Ibid. - P. 40.

27. Ibid. - P. 46.

28. The discovery of the accelerated expansion of the universe. Nobel Prize winners in physics for 2011.

29. Lazarev A.S. The deciphered Bible, or the requiem of civilization. - M.: ASU, 2002. - P. 16.

30. Panasiuk B. Man, nature and the universe (the solution of secrets). - M.: Parapan, 2008. - P. 272.

31. Ibid. - P. 130.

32. Ibid. - P. 249.

33. Ibid. - P. 132.

34. Ibid. - P. 269.

35. Agricultural Biology. Series: Plant Biology. - M., 2013. - No. 5. - P. 26.

36. Soviet encyclopedic dictionary. Ed. The fourth. - M.: Soviet Encyclopedia, 1989. - P. 1443.

37. Ibid. - P. 975.

38. Timiryazev K. Life of plants. Fav. op. - M.: Selhozizdat, 1949. - T. 3. - P. 164.

39. Ibid.

40. Ibid. - P. 172. 